Z Herz- Thorax- Gefäßchir 2020 · 34:214-216 https://doi.org/10.1007/s00398-020-00368-0 Online publiziert: 14. Mai 2020

(c) Springer Medizin Verlag GmbH, ein Teil von Springer Nature 2020

Die „blue baby operation“, die erstmals im November 1944 am Johns Hopkins Hospital in Baltimore durchgeführt wurde, ist wie wohl kein anderer Eingriff zum Symbol dafür geworden, dass die Herzmedizin die Schwelle in eine andere Dimension überschritten hatte. Hierfür bedurfte es allerdings des Aufeinandertreffens dreier Menschen und Charaktere, wie sie wohl unterschiedlicher kaum hätten sein können: des Chirurgen Alfred Blalock, seines technischen Assistenten Vivien Thomas und der Kinderkardiologin Helen Taussig (Portrait in Z Herz- Thorax- Gefäßchir 2019, Ausgabe 3, S. 224-225).

Alfred Blalock (• Abb. 1) wurde am 05.04.1899 in Culloden, Georgia, USA, geboren, einem Ort mit damals 334 Einwohnern, als das erste von 5 Kindern einer Familie aus einer der großen Baumwolldynastien in den Südstaaten. Schon früh entwickelte er das charakteristische Bild eines Südstaatlers mit einem smarten Wesen, einem gepflegten, attraktiven Äußeren, einer ruhigen, sanften Sprache, aber durchaus in der Lage, hiermit auf eine effektive Weise seine Meinung durchzusetzen. Er selbst und andere hielten ihn zwar nicht für einen ausgesprochen fleißigen, aber für einen intelligenten und „sozial umgänglichen“ Schüler und Studenten. Noch während

Dieser Beitrag ist eine aktualisierte Fassung des Kapitels "Meilensteine der Entwicklung: Alfred Blalock und Vivien T Thomas" in: Weil J, Kallfelz HC, Lindinger A, Schmaltz AA (Hrsg) (2019) Kinderkardiologie in Deutschland: 50 Jahre Deutsche Gesellschaft für Pädiatrische Kardiologie 1969-2019; Elsevier, S. 345-347 (mit freundlicher Genehmigung des Elsevier Verlages).

\author{
H. E. Ulmer \\ Heidelberg, Deutschland
}

\title{
Alfred Blalock, Vivien Thomas, Helen Taussig und die „blue baby operation"
}

seiner Studienzeit musste er sich in 1921 wegen einer Hydronephrose einer linksseitigen Nephrektomie unterziehen. Dies vermochte ihn jedoch nur vorübergehend von einem aktiven Clubleben an der Universität als Tennischampion, erfolgreicher Golfer und aktiver „ladies' man" abzuhalten. Da sein Abschluss an der University of Maryland in Baltimore in 1922 nicht ausreichend war, um eine seinen Wünschen entsprechende chirurgische Stelle am Johns Hopkins Hospital zu erringen, ging er 1925 zusammen mit seinem lebenslangen engen Freund Tinsley Harrison, dem späteren Herausgeber des weltbekannten Lehrbuchs Harrison's principles of internal medicine als erster chirurgischer Resident an die damals neu ausgerichtete Medical School der Vanderbilt University in Nashville, Tennessee. Seinen ausgeprägten wissenschaftlich orientierten Interessen folgend, konnte er dort ein eigenes chirurgisches Labor zur Erforschung der Ursachen und Behandlung des traumatischen Schocks etablieren. Die segensreichen und v.a. praktisch anwendbaren Ergebnisse machten ihn rasch bekannt und halfen, vielen Menschen das Leben $\mathrm{zu}$ retten.

Das Auftreten einer schweren Lungentuberkulose mit wiederholten Eingriffen und Sanatoriumsaufenthalten, u. a. auch in der Schweiz, unterbrach seine Karriere für 2 lange Jahre, bis er 1928 wieder nach Nashville zurückkehren und seine Arbeit fortsetzen konnte. Das Jahr 1930 brachte ihn dann mit zwei, sein weiteres Leben bestimmenden Menschen zusammen. Am 01.01. stellte er Vivien Thomas, einen farbigen „housekeeper“, als seinen persönlichen Laborhelfer ein, und in der
Verwaltung der Universität begegnete er Mary Chambers O’Bryan, einer ausgewiesenen Südstaatenschönheit, die er im Oktober 1939 heiratete.

Fachlich und rhetorisch begabt, entwickelte sich Blalock zunehmend zu einem mutigen und innovativen Chirurgen und Lehrer. 1938 wurde er zum Professor of Surgery der Vanderbilt University ernannt. Wissenschaftlich befasste er sich in engster Zusammenarbeit mit dem sich in chirurgischen Techniken als außerordentlich talentiert erweisenden Vivien Thomas inzwischen mit der Erstellung eines Tiermodells zur Erzeugung einer pulmonalen Hypertension durch verschiedene intrathorakale Gefäßanastomosen. Es gelang ihnen zwar, einen höheren pulmonalen Blutfluss, aber keine Druckerhöhung zu erzeugen.

1941 wurde Alfred Blalock, nach Absagen mehrerer anderer Kandidaten, und nicht ohne Widersprüche, zum Chairman des Department of Surgery der Johns Hopkins University in Baltimore gewählt - dem Lehrstuhl, an dem er 16 Jahre zuvor als Assistent nicht angenommen worden war. Eine seiner Bedingungen war die Übernahme seines großen chirurgischen Forschungslabors, einschließlich dessen technischen Leiters, des Farbigen Vivien Thomas.

Vivien Theodore Thomas (- Abb. 2) wurde am 29.08.1910 in New Iberia, Louisiana, USA, als Enkel eines ehemaligen Sklaven geboren. So konnte er zunächst auch die „Cotton-Picking High School“ besuchen. Nach der Weltwirtschaftskrise musste er jedoch seine Hoffnung auf eine weitere Ausbildung aufgeben und die Stelle einer Reinigungskraft in den experimentellen Labors der 


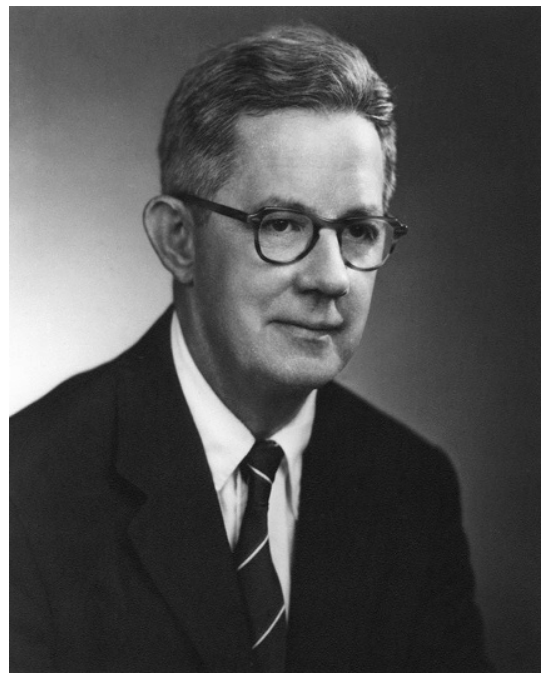

Abb. 1 A Alfred Blalock (1899-1964). (Mit freundlicher Genehmigung von $\odot$ Medical Archives of the Johns Hopkins Medical Institutions. Alle Rechte vorbehalten)

Vanderbilt University annehmen. Dort begegnete er 1930 Alfred Blalock, dem sein sorgfältiger Umgang mit den Geräten im Labor aufgefallen war. Blalock bildete Thomas zu seinem zunehmend unverzichtbaren Laborgehilfen aus, der bald alle erforderlichen chirurgischen Techniken besser beherrschte als der Chirurg selbst. Dies führte dazu, dass Blalock ein neues chirurgisches Experiment zwar konzipierte, dieses dann aber von Thomas vom praktischen Tierversuch bis zur Auswertung der Daten selbstständig durchgeführt wurde. Thomas wurde, wie sich bald herausstellen sollte, für Blalock im Labor unersetzlich, sichtbar an einer mehr als 3 Jahrzehnte andauernden erfolgreichen Zusammenarbeit. Aufgrund der unterschiedlichen rassischen Herkunft konnte zur damaligen Zeit jedoch zwischen den beiden Männern keine soziale Freundschaft entstehen. So durfte Thomas z.B. seine Arbeitsstätte, das Gebäude der Universität, nie durch den Haupteingang, sondern immer nur über die Rückseite betreten.

Helen Brooke Taussig (• Abb. 3), geboren im Mai 1898 in Cambridge, Massachusetts ( $\mathrm{Z}$ Herz- Thorax- Gefäßchir 2019, Ausgabe 3, S. 224-225) war, den Gegebenheiten folgend, um als Frau

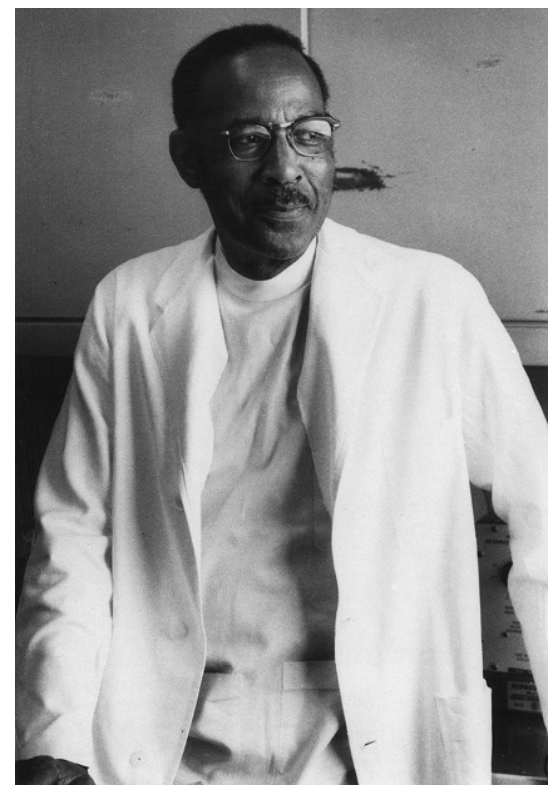

Abb. 2 ム Vivien Thomas (1910-1985). (Mit freundlicher Genehmigung von $\odot$ Medical Archives of the Johns Hopkins Medical Institutions. Alle Rechte vorbehalten)

praktisch in der Medizin tätig sein zu dürfen, 1923 gezwungenermaßen nach Baltimore gekommen. Ihr von Anfang an bestehendes Interesse für angeborene Herzfehler hatte sie 1930 dort zur Leiterin eines eigens hierauf spezialisierten Bereichs, der „Harriet Lane Clinic“ der Johns Hopkins Universität gemacht. Mit der ersten erfolgreichen Ligatur eines persistierenden Ductus arteriosus durch den Chirurgen Robert Gross 1938 in Boston begannen sich damals, neue therapeutische Möglichkeiten für angeborene Herzfehler abzuzeichnen. Nach Helen Taussigs Erfahrungen überlebten Kinder mit einer Fallot-Tetralogie bei zusätzlicher Persistenz eines Ductus arteriosus deutlich länger als die anderen „blue babies“. Sie verfolgte daher die Vorstellung, durch operatives Anbringen eines künstlichen Ductus vielen dieser Kinder helfen zu können. Als sie dieses Vorhaben 1942 dem Chirurgen Robert Gross vortrug, lehnte dieser den vorgeschlagenen Eingriff brüsk ab.

1943 nahm Helen Taussig dann erstmals von den chirurgischen Möglichkeiten im eigenen Hause durch Alfred Blalock Kenntnis. Mit dem smarten Südstaatler hatte die groß gewachsene, zarte Lady mit ihrem gepflegten New-England-Akzent bis dahin kaum Kontakt ge-

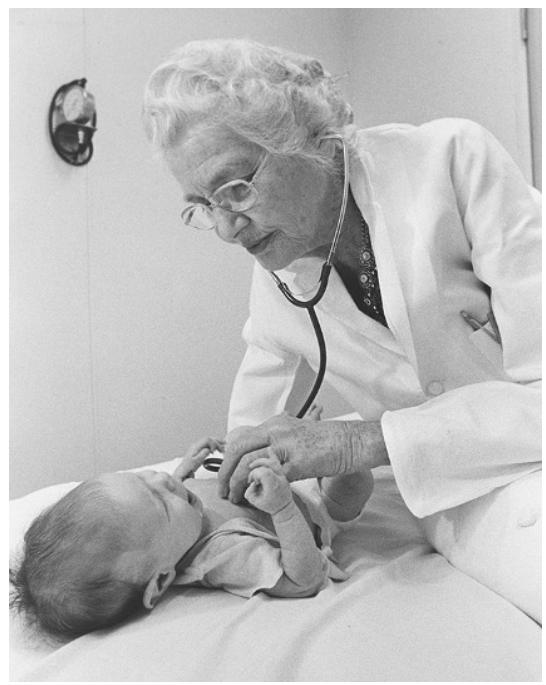

Abb. 3 ム Helen Taussig (1898-1986). (Mit freundlicher Genehmigung von $\odot$ Medical Archives of the Johns Hopkins Medical Institutions. Alle Rechte vorbehalten)

habt, bis sie von seinen früheren Erfahrungen mit aortopulmonalen Shunts im Tierversuch erfuhr. Auf ihr Drängen hin nahmen Blalock und v. a. Thomas diese Versuche erneut auf, wenn auch nun mit einer anderen Zielsetzung.

Die chirurgische Technik der Operation, zwischen der A. subclavia und einem Ast der Pulmonalarterie eine Anastomose herzustellen, hatte sich nach mehr als 100 Experimenten im Labor zunehmend als machbar erwiesen, als das weitere Vorgehen eine dramatische Entwicklung nahm. Unter den Patienten Helen Taussigs befand sich die inzwischen 15 Monate alte und dabei nur $4,5 \mathrm{~kg}$ schwere Eileen Saxon, die sich wegen ihres Herzfehlers, einer Fallot-Tetralogie, mit schwerer Zyanose seit ihrem 4. Lebensmonat unter einem Sauerstoffzelt in der Klinik befand. Im November 1944 hatte die Versorgung ihres Blutes mit Sauerstoff derart lebensbedrohliche Ausmaße angenommen, dass nahezu täglich mit ihrem Ableben gerechnet werden musste. Daher wurde notfallmäßig der 29.11.1944 für den ersten klinischen Einsatz der bis dahin nur experimentell erprobten Operation festgesetzt.

Am frühen Morgen wurde Eileen in den OP-Raum 706 des John Hopkins Hospitals gebracht. Die Narkose wurde 


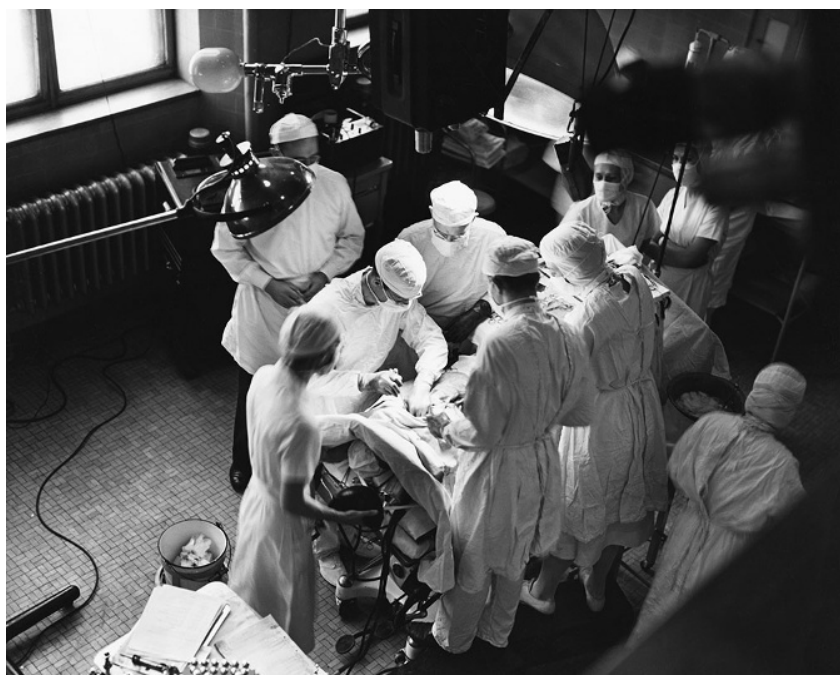

Abb. $4<$ „The blue baby operation", 29.11.1944. (Mit freundlicher Genehmigung von $(\odot$ Medical Archives of the Johns Hopkins Medical Institutions. Alle Rechte vorbehalten)

von Marel Harmel mit getropftem Äther und Beutelbeatmung mit der Hand durchgeführt. Blalock als Operateur stand linker Hand sein 1. Assistent William Longmire (später UCLA) zur Seite, der zweite Assistent Denton Cooley (später Texas Heart Institute, Houston) stand ihm gegenüber, durch seine Körperlänge kaum zu verkennen, rechts neben ihm Helen Taussig (• Abb. 4). Obwohl alle bereitstanden, weigerte sich Blalock, mit der Operation zu beginnen, bevor nicht Vivien Thomas, der erst aus dem Labor geholt werden musste, unmittelbar hinter ihm stand. Blalock selbst hatte die Operation zuvor erst ein einziges Mal im Tierversuch gemacht, im Gegensatz zu Thomas, der nach Hunderten von Experimenten mit jedem Handgriff bestens vertraut war. Praktisch gesehen ließ sich Alfred Blalock bei Eileens Operation von Vivien Thomas verbal die Hand führen. Nach einer linksseitigen Thorakotomie wurde die linke A. subclavia mit dem dünnen linksseitigen Pulmonalisast anastomosiert. Die Operation dauerte zwei Stunden. Die Zyanose besserte sich, und Eileen überlebte. Die nächsten beiden Wochen waren durch wiederholte Pneumothoraces und eine pulmonale Infektion erschwert. Danach verbesserte sich der Allgemeinzustand des Mädchens so deutlich, dass sie Anfang Januar 1945 nach Hause entlassen werden konnte. Im Laufe des Jahres nahm die Zyanose jedoch erneut wieder $\mathrm{zu}$, sodass der gleiche Eingriff bei Eileen am 01.08.1945, nun auf der rechten Seite, noch einmal durch- geführt werden musste. Diese Belastung war für den geschwächten Körper des Mädchens zu viel. Eileen Saxon verstarb am 5. postoperativen Tag.

Das Prinzip der Operation, die „Blalock-Taussig-Anastomose“, hatte sich jedoch als richtig und machbar erwiesen. Die beiden nächsten Patienten, die schon im Februar 1945, kurz nach Eileen, erfolgreich operiert worden waren, überlebten etliche Jahre. Im Jahr 1945 operierte die Gruppe in Baltimore 247 Kinder mit einer Letalität von nur $23 \%$.

Die Publikation der ersten 3 Fälle erfolgte unter den Autoren Alfred Blalock und Helen Taussig und erschien bereits am 19.05.1945 im Journal of the American Medical Association (JAMA). Der entscheidende Anteil von Vivien Thomas wurde darin mit keinem Wort erwähnt. Das Johns Hopkins Hospital, das sich bis dahin von jeglicher $\mathrm{Pu}$ blicity fern gehalten hatte, wurde nun plötzlich zu einem Zentrum der kardiologischen Welt. Es gab kein Land der Erde, aus dem nicht wenigstens einige Ärzte nach Baltimore kamen, um sich vor Ort zu informieren. 1947 erlebten Alfred Blalock und Helen Taussig einen wahren Triumphzug anlässlich einer Einladungsreise nach London, Stockholm und Paris, um die Operation in Europa einzuführen. Im Guy's Hospital in London operierte Blalock 10 „blue babies" in Folge ohne einen einzigen Todesfall. Als er im Royal College of Surgeons einen Vortrag hielt, standen die Leute bis auf die Straße Schlange, und die „Show“ musste am nächsten Tag noch einmal wiederholt werden.

An der kommenden Entwicklung der Chirurgie angeborener Herzfehler, wie später z. B. mit der Herz-Lungen-Maschine, hatte zwar die Johns Hopkins Universität weiteren Anteil, Alfred Blalock selbst hat jedoch nie eine Operation am offenen Herzen durchgeführt.

Die Vielzahl von Auszeichnungen, Präsidentschaften und Ehrenmitgliedschaften von Universitäten aus den Vereinigten Staaten und Europa, die Alfred Blalock zuteilwurden, ist kaum zu benennen. Dennoch blieb er unverändert seiner Arbeit am Johns Hopkins Hospital der School of Medicine in Baltimore bis zu seiner Emeritierung im Mai 1964 treu. Nur wenige Monate später, am 15.09.1964, erlag er einem Urogenitalkarzinom, wohl einer Spätfolge seiner in den 1920er-Jahren durchgemachten Grunderkrankung in diesem Bereich.

Vivien Thomas blieb nach Blalocks Tod noch 15 Jahre auf seiner Laborstelle tätig. Er begann damit, in dem Labor, dessen Direktor er inzwischen geworden war, andere Techniker, aber auch Chirurgen, wie z. B. Denton Cooley, in seiner Operationsmethode und anderen chirurgischen Techniken praktisch auszubilden. 1976 verlieh ihm die Johns Hopkins Universität eine Ehrendoktorwürde, allerdings nicht in Medizin, sondern in den Rechten, da er keinen medizinischen akademischen Grad aufzuweisen hatte. Dennoch wurde er gleichzeitig in den Lehrkörper der Medical School aufgenommen und sein Portrait im Foyer neben dem Alfred Blalocks aufgehängt. Thomas schrieb eine später sehr bekannte Autobiographie Partners of the heart, die auch verfilmt wurde (Something the Lord made/Ein Werk Gottes, 2004). Er verstarb im November 1985 an einem Pankreaskarzinom, wenige Tage vor Erscheinen seines Buches.

\section{Korrespondenzadresse}

\section{Prof. Dr. H. E. Ulmer}

Am Aukopf 5, 69118 Heidelberg, Deutschland herbert_ulmer@t-online.de 